\title{
Advances in the understanding of
}

\section{nanomaterial-biomembrane interactions and their}

\section{mathematical and numerical modeling}

The widespread application of nanomaterials (NMs), which has accompanied advances in nanotechnology, has increased their chances of entering an organism, for example, via the respiratory system, skin absorption or intravenous injection. Although accumulating experimental evidence has indicated the important role of NM-biomembrane interaction in these processes, the underlying mechanisms remain unclear. Computational techniques, as an alternative to experimental efforts, are effective tools to simulate complicated biological behaviors. Computer simulations can investigate NM-biomembrane interactions at the nanoscale, providing fundamental insights into dynamic processes that are challenging to experimental observation. This paper reviews the current understanding of NM-biomembrane interactions, and existing mathematical and numerical modeling methods. We highlight the advantages and limitations of each method, and also discuss the future perspectives in this field. Better understanding of NM-biomembrane interactions can benefit various fields, including nanomedicine and diagnosis.

\section{KEYWORDS: biomembrane interaction mathematical modeling nanomaterial numerical modeling}

With the rapid development of nanotechnologies, nanomaterials (NMs) have become part of our daily lives. They are used in cosmetics, textiles, bioimaging, medicine and diagnosis $[1-8]$. The widespread applications of NMs increase their chances of entering the human body via a number of pathways, such as the respiratory system [9], skin absorption [10,11], intravenous injection [12] and implantation [13]. As a result, there is an urgent need to understand the potential physiological and pathological reactions after exposure to NMs [14,15]. NMs can induce a series of complicated reactions, but they first need to cross a biomembrane barrier (e.g., skin, lung surfactant, intestinal barrier or cell membrane) to reach target cellular compartments [16]. Therefore, it is of great importance to investigate NM-biomembrane interactions.

Significant efforts have been devoted to experimentally investigating NM-biomembrane interactions. Many important findings have been obtained from these efforts. For instance, it has been established that NMs can induce specific interactions with targeted cell membranes via specific ligands [17], modify the biconcave shape of erythrocytes [18], change cell membrane asymmetry and thickness $[19,20]$, damage the integrity of cells through hole formation [21] and induce oxidative stress [18]. In addition, NMs can be rapidly taken up by cells; a characteristic that has been applied for cancer gene therapy [22,23], cancer imaging and phototherapy [24], and drug delivery $[25,26]$. However, despite all of these important applications, the mechanisms underlying NM-biomembrane interactions are poorly understood, as experimental tests and observations at the nanoscale for complex biological systems remain technically challenging.

Alternatively, it has been demonstrated that mathematical and numerical modeling can simulate the NM-biomembrane interaction at the nanoscale, as well as conditions that are challenging to realize experimentally, providing deep insight into the dynamic process of the system [27-29]. Moreover, the capability of numerical simulations has been significantly enhanced with the increase in computing power in the last few decades. In this review, we present the state-of-the-art advances in the understanding of NM-biomembrane interactions and their mathematical and numerical modeling. The 'Pathway of cellular uptake of NMs' and 'Key factors for NM-biomembrane interactions' sections describe the basic cellular uptake pathways of NMs and key factors associated with NM-biomembrane interactions, respectively. The 'Mathematical \& numerical modeling approaches for NM-biomembrane interactions' section reviews the existing mathematical and numerical modeling approaches for NM-biomembrane interactions and discusses the advantages and disadvantages associated with each method. Finally, the 'Discussion' and 'Conclusion \& future perspective' sections highlight the limitations of the

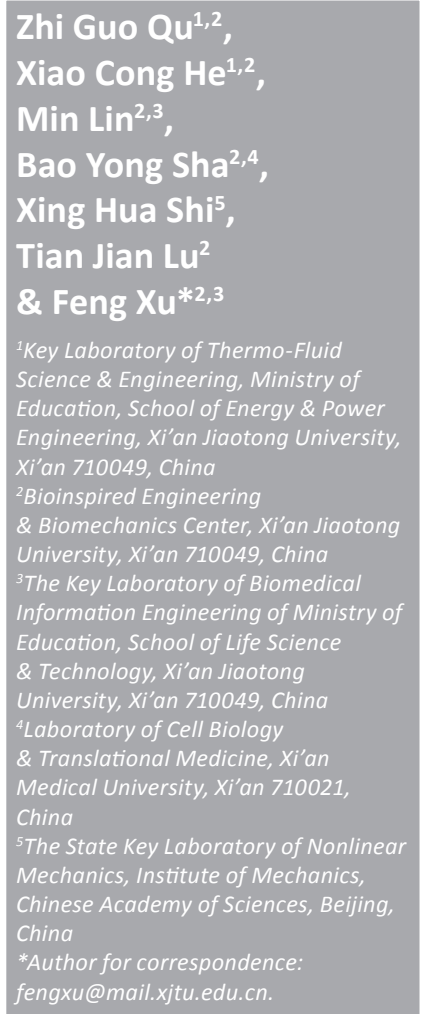

Future $\mathrm{fSS}$ 
current modeling methods and future research topics for NM-biomembrane interactions.

\section{Pathways of cellular uptake of NMs}

The cellular uptake of NMs usually consists of two processes, adsorption of NMs on the membrane, and internalization of NMs by endocytic and nonendocytic pathways (FIgure 1) [16,30-33].

\section{Endocytic pathway}

Endocytosis is an important pathway for cellular uptake because the most important substances, such as proteins, which are biomacromolecules, cannot directly penetrate the cell membrane. Endocytosis can be divided into phagocytosis and nonphagocytosis; the latter includes macropinocytosis, clathrin-mediated endocytosis (CME), caveolae-mediated endocytosis (CvME), and other clathrin- and caveolae-independent endocytosis pathways [34]. CME is the predominant receptor-mediated endocytosis pathway for most cells, and involves membrane curvature generation, clathrin lattice formation and clathrin-coated vesicle formation. CvME forms flask-shaped invaginations in the membrane that allow molecules to enter the cells through ligand-receptor binding [35]. Other types of clathrin- and caveolae-independent endocytic pathway have been discovered aside from CME and CvME, such as lipid raft-mediated and ARF6-mediated GEEC pathways [36].

A variety of NMs (e.g., carbon nanotubes [CNTs], metal NMs and semiconductor NMs) have been demonstrated to enter into the cells via endocytosis [37-44]. For instance, both singlewalled nanotubes (SWNTs) and SWNT conjugates (SWNT-streptavidin, protein-SWNT and DNA-SWNT) can enter human promyelocytic leukemia (HL60) cells via endocytosis $[39,40]$. Several factors affect the endocytosis process [45], such as NM size, membrane elasticity and receptor-ligand interactions. For instance, most nanosized synthetic NMs are internalized through endocytosis [35], and the optimal size of NMs for endocytosis is $25-30 \mathrm{~nm}[46,47]$. An increased membrane elasticity can reduce the membrane bending energy, enhancing the ability of the membrane to wrap around NMs [48]. In addition, specific receptor-ligand interactions are involved in the endocytosis process (Figure 2), which is the most effective promotive force for endocytosis [45].

\section{Nonendocytic pathways}

Although endocytosis is the main pathway for cellular uptake of NMs, nonendocytic pathways exist, where NMs directly penetrate the cells [35]. For instance, NMs with sufficiently small diameters (e.g., metal clusters [49]) and needle-shaped NMs (e.g., single CNTs [50]) can directly penetrate the membrane. Cationic NMs (e.g., PAMAM dendrimers [51,52], $\mathrm{Au}-\mathrm{NH}_{2}$ and $\left.\mathrm{SiO}_{2}-\mathrm{NH}_{2}[17]\right)$ can pass through the membrane by forming pores in the membrane. Large NMs can also penetrate into the cells when attached to cell-penetrating peptides (CPPs) [33,53]. CPPs

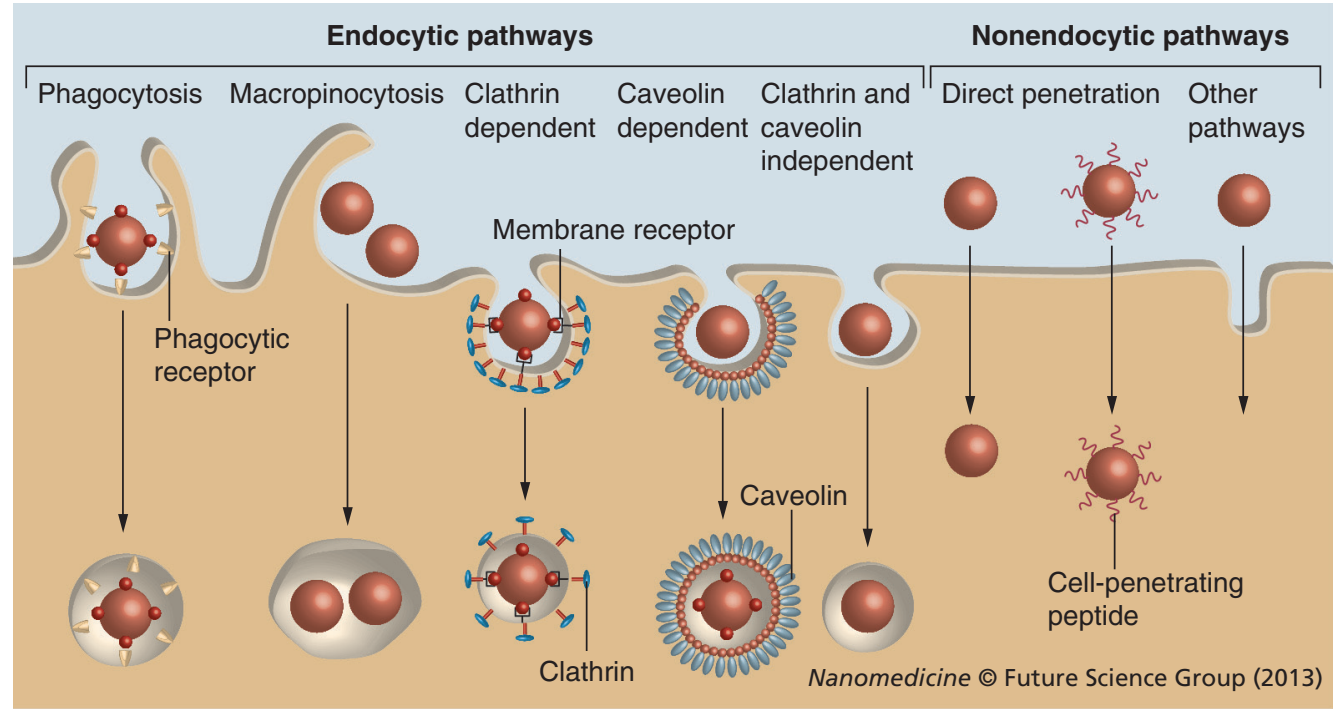

Figure 1. Cellular uptake pathways of nanomaterials. The cellular uptake pathways of nanomaterials can be simply divided into two kinds, namely, endocytic and nonendocytic. The endocytic pathway includes phagocytosis, macropinocytosis, clathrin-dependent endocytosis, caveolae-dependent endocytosis, and other mechanisms of clathrin- and caveolae-independent endocytosis. The nonendocytic pathway includes direct penetration and other pathways, which are not yet fully understood. 


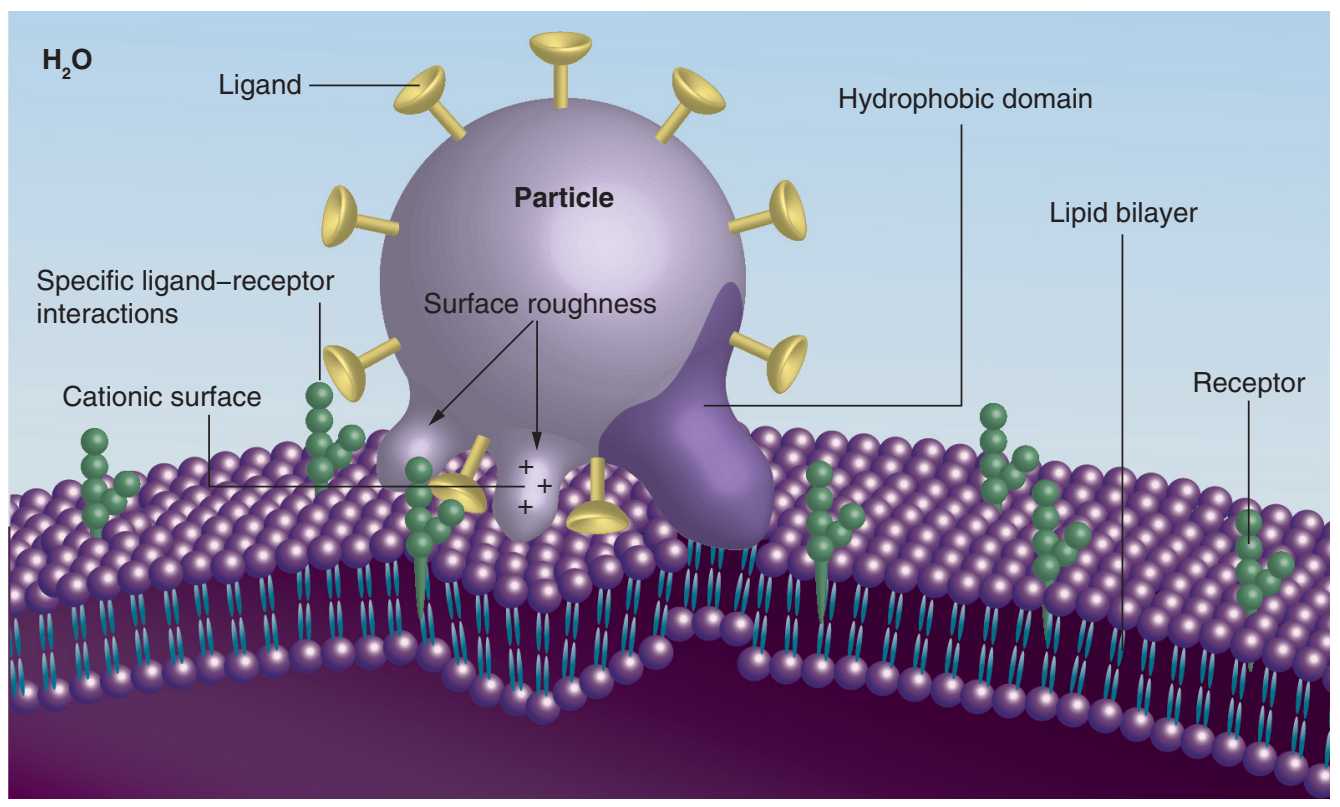

Figure 2. Receptor-mediated cellular uptake of nanomaterials. The interactions between nanomaterials and the biomembrane can be classified into two kinds: specific receptor-ligand interactions and nonspecific interactions. Specific receptor-ligand interactions always lead to endocytosis. The nanomaterial properties (e.g., surface roughness, hydrophobicity and charge) that promote binding with lipid molecules generally relate to nonspecific forces, such as hydrophobic and electrostatic interactions. The nonspecific interactions can lead to direct penetration of the membrane.

Reproduced with permission from [45].

can effectively deliver nanomedicine to the intercellular target by passing through the plasma membrane [54]. Studies have found that peptideencapsulated fluorescent silver nanoclusters [49] and polyarginine-conjugated iron oxide NMs loaded with siRNA can directly penetrate cell mebranes [55]. Similar to CPPs, several synthetic NMs, such as synthetic amphipathic phospholipid polymers [56] and nanoparticles (NPs) coated with hydrophilic-hydrophobic striated ligand shell [57,58] can also penetrate the membrane in a similar manner to the CPPs without disrupting the membrane.

TABLE 1 summarizes the cellular uptake pathways of different NMs. Although numerous studies have been performed, as previously mentioned, the entry mechanism of NMs through the membrane is far from clear. For instance, no precise definition exists for different entry pathways of NMs into cells. More work is needed to better understand the NM-biomembrane interactions.

\section{Key factors for NM-biomembrane interactions \\ - Physicochemical properties of NMs}

Key physicochemical properties of NMs which affect NM-biomembrane interactions include NM size, shape, surface roughness and rigidity, charge, hydrophobic properties, and protein corona.

NM size can determine the entry pathways of the NMs into the cells and affect the rates of cellular uptake. For instance, silica NMs with a size of less than $1.2 \mathrm{~nm}$ can penetrate into a dimyristoylphosphatidylcholine lipid bilayer without damaging the membrane, while pores form in the lipid membrane for NMs with a size ranging from 1.2 to $22 \mathrm{~nm}$, and the membrane envelopes NMs with a size over $22 \mathrm{~nm}$ (Figure 3) $[59,60]$. The modes of endocytosis depend on the NM size, which is typically $100 \mathrm{~nm}$ for CME and 50-80 nm for CvME, respectively [34]. Moreover, studies have found that the cellular uptake rate for gold (Au)-NMs with sizes of approximately $50 \mathrm{~nm}$ is faster than that of other sizes [37,61].

NM shape is another important factor that affects the cellular uptake process. For instance, the polystyrene NM shape determines the formation of the actin structures that initiate phagocytosis [62]. The endocytosis rate of spherical Au-NMs (74 and $14 \mathrm{~nm}$ in diameter) was higher than that of rod-shaped Au-NMs of the same size in one study. Furthermore, the endocytosis rate of rod-shaped Au-NMs $(74 \times 14 \mathrm{~nm})$ with a low aspect ratio (1:3) was greater than the high-aspect-ratio (1:5) NMs [37]. However, in another study, the rate of cellular uptake of 
REVIEW Qu, He, Lin et al.

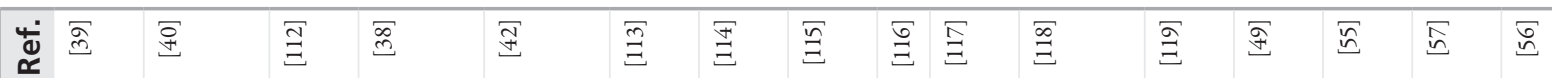
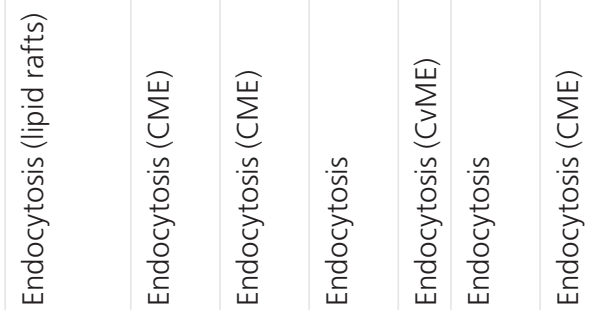

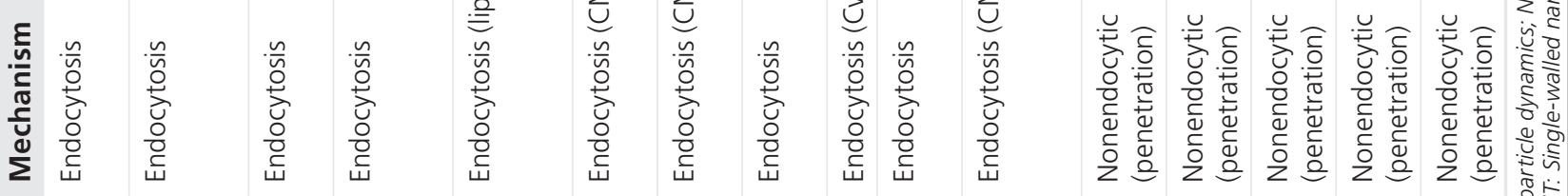

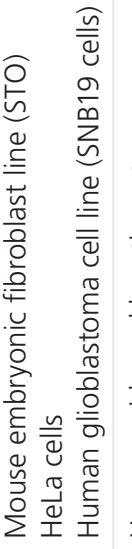

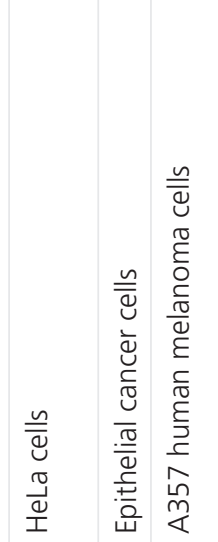

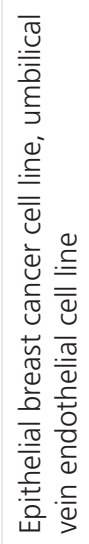
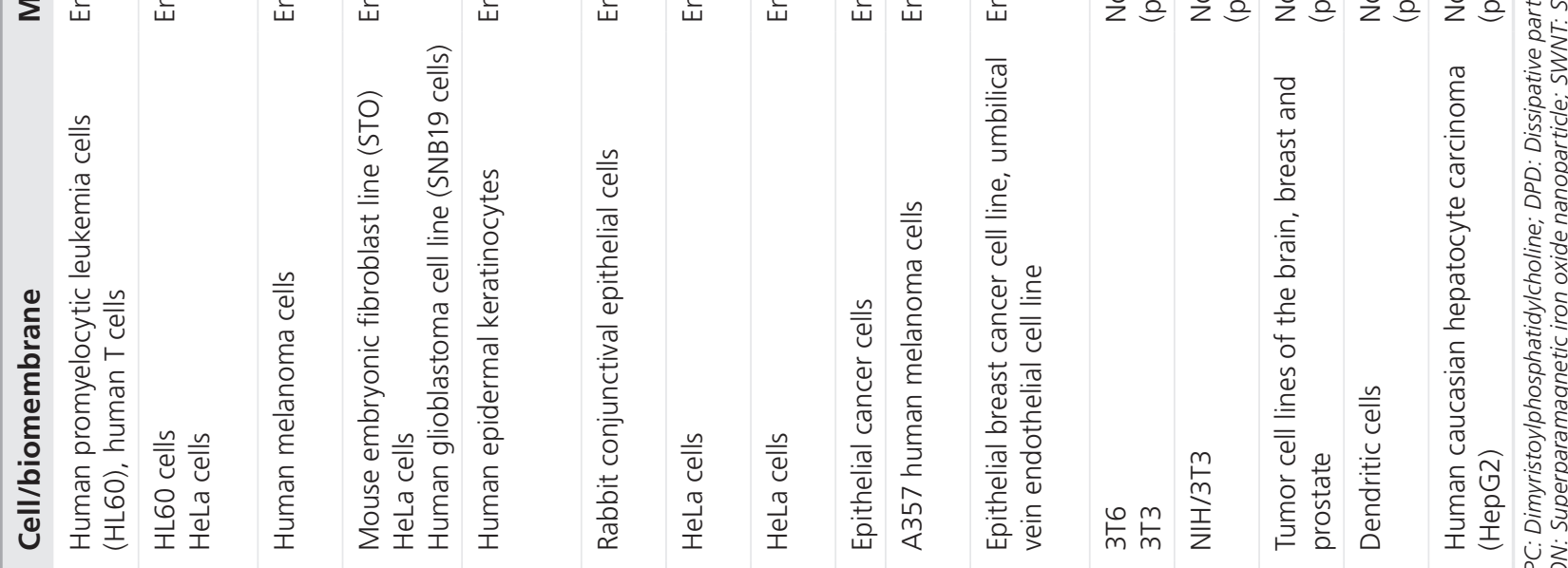

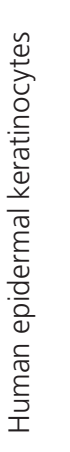
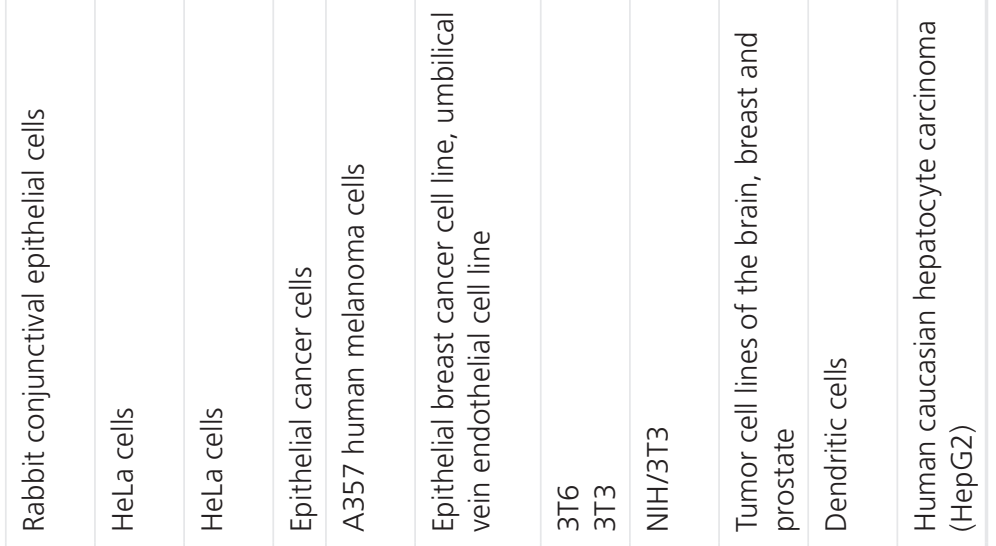

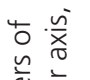

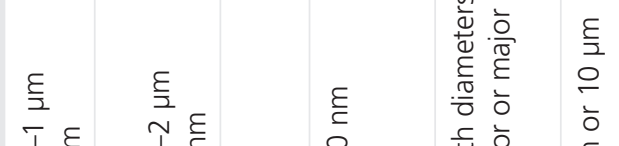

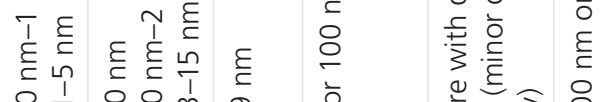

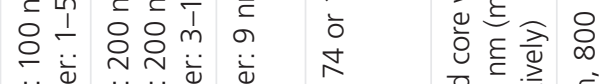

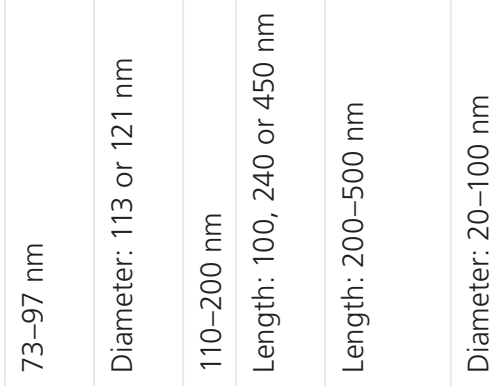

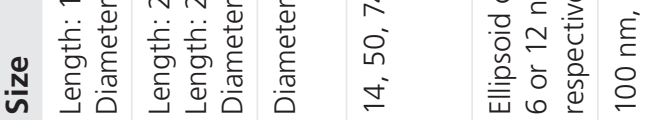

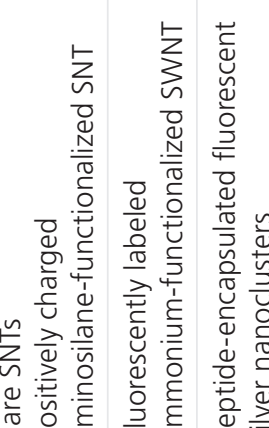

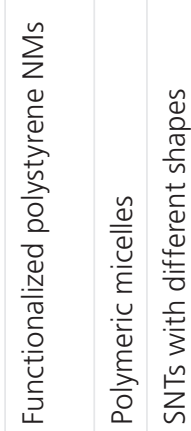

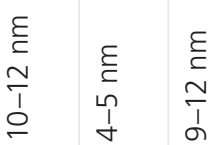

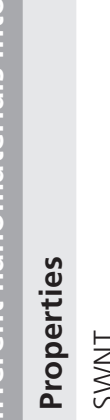

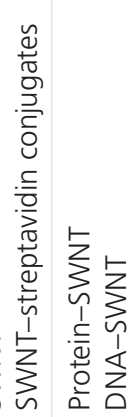

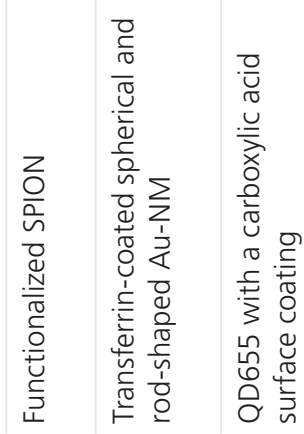

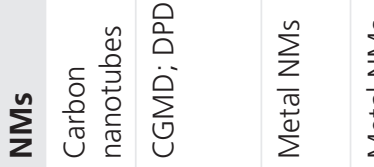

$\sum_{\substack{\frac{n}{\pi} \\ \frac{\pi}{0}}}^{\infty}$

\&

喜喜量

¿ छ

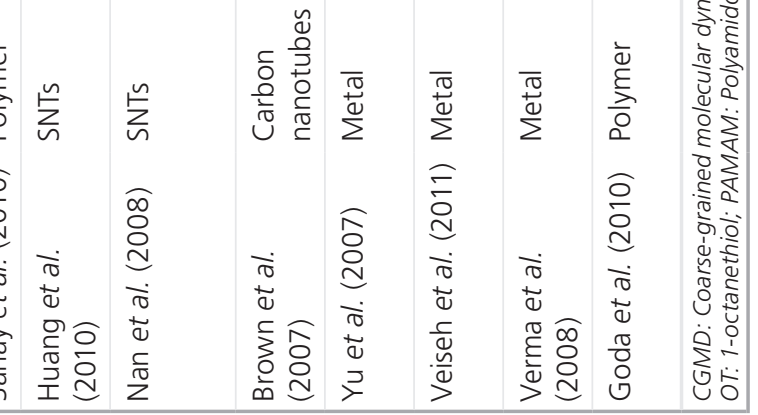




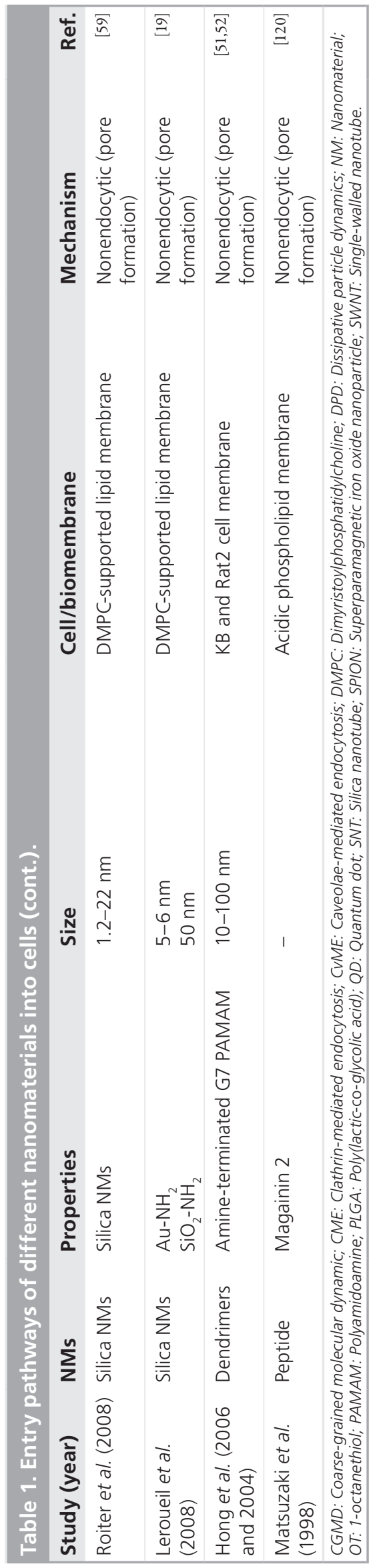

high-aspect-ratio cylindrical hydrogel particles (diameter: $150 \mathrm{~nm}$; height: $450 \mathrm{~nm}$ ) into HeLa cells was four-times faster than that of lowaspect-ratio cylindrical NMs (diameter: $200 \mathrm{~nm}$; height: $200 \mathrm{~nm}$ ) with a similar volume. One possible explanation is that the high-aspect-ratio cylindrical particles have larger surface areas to contact the membrane [63]. Numerical simulations have also revealed that the minimum driving forces for NMs to penetrate the membrane depend on the NM shape (e.g., sphere, ellipsoids, rods, discs and pushpin-like) [64]. Shi et al. simulated the entry mechanism of $1 \mathrm{D}$ CNTs with different tips (hemispherical cap, enlarged cap, CNT bundle tip and open tip) into cells and found that different tips resulted in different cell entry modes [65].

Surface roughness and rigidity of NMs also affect the NM-biomembrane interactions. For instance, silica NMs with a smooth surface (22 $\mathrm{nm}$ in diameter) were completely enveloped by the lipid membrane, whereas the bumpy NMs with the same size were only partially enveloped, as shown in Figure 3 [60]. In the study of Shi et al., soft NMs were more difficult to be fully wrapped by the membrane than rigid NMs, because a larger adhesion energy was needed for the former [66].

Electrostatic interactions and hydrophobic interactions are the two main nonspecific interaction types in NM-biomembrane interactions. Negatively charged cell plasma membrane results in a greater internalization potential of positively charged NMs relative to neutral and negatively charged NMs [41,67], because the electrostatic attraction promotes the adsorption of positively charged NMs on the membrane. Both experimental studies and computer simulations indicated that cationic Au-NMs induce more disruption of the cell membrane than anionic Au-NMs [68,69], as it depends on the charge density. For the hydrophobic interactions, a simulation study showed that hydrophobic NMs (diameter: $10 \mathrm{~nm}$ ) could be embedded into the cell membrane, whereas semihydrophilic NMs only adhered to the membrane [70]. 11-mercapto-1-undecanesulfonate Au-NPs that only had hydrophilic sulfonate ligands were endocytosed by cells, whereas the 66-34 1-octanethiol Au-NPs (Au-NPs coated with a 2:1 molar mixture of 11-mercapto-1-undecanesulfonate and 1-octanethiol) with a hydrophilic-hydrophobic striated ligand shell directly penetrated the cell membrane owing to ligand rearrangement [57].

Before the NMs get close to the membrane surface, such as in the blood, plasma 


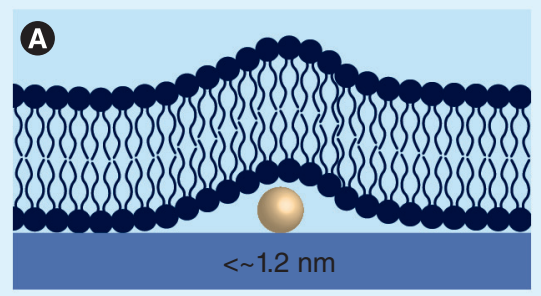

\section{B}

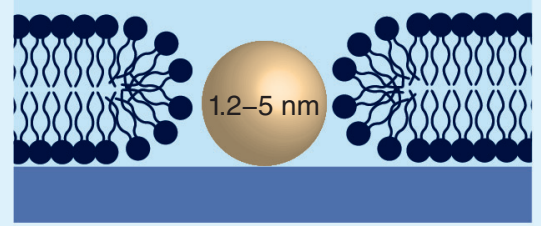

$\boldsymbol{0}$

\begin{abstract}
(D)
\end{abstract}
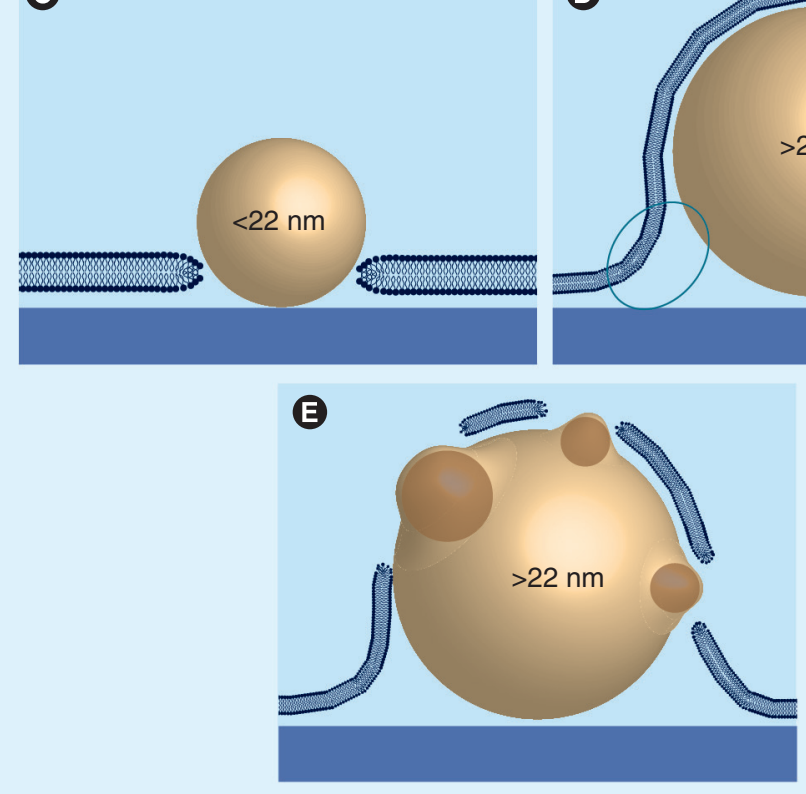

Figure 3. Interaction mechanisms of silica nanomaterials of different sizes with supported lipid membranes. (A) Nanomaterials (NMs) with sizes smaller than $1.2 \mathrm{~nm}$ penetrated into the lipid membrane without disrupting it. (B \& C) The NMs with a size of 1.2-22 $\mathrm{nm}$ could form pores in the lipid membrane, (D) whereas NMs with a size over $22 \mathrm{~nm}$ were enveloped by the lipid membrane. (E) The bumpy NMs (>22 nm) were partially enveloped.

Reproduced with permission from [59,60]. phosphatidylethanolamine depending on their hydrophilic head groups. Phosphatidylcholine and phosphatidylethanolamine are neutrally charged, while phosphatidylserine is negatively charged. In general, the cell membrane is electronegative and will repel negatively charged and attract positively charged NMs [75]. The membrane structure (e.g., phase transition and heterogeneity of the membrane) is also a key factor. For instance, the polycationic polymer NMs disrupt the lipid membrane in a membrane structure-dependent manner, with only the liquid-crystalline phase found to induce disruption [76]. With proteins, sugar and cholesterol on it, the membrane always presents heterogeneity. The interaction of NPs and the heterogeneous membrane with an adhesive raft on the membrane was simulated in one study, where the raft drove the entry process of membrane-wrapped NPs into the vesicle [77].

The properties of biomembranes are significantly dependent on environmental factors, such as temperature, $\mathrm{pH}$ and salt concentration [78-80]. For instance, the membrane fluidity decreases and changes from the liquid-crystalline to gel phase with decreasing temperature, which inhibits NM-biomembrane interactions $[76,80]$. The environmental $\mathrm{pH}$ and salt concentration can alter the mechanical and electrical properties of the membrane. The compressive modulus of the PC membrane was reduced by $30 \%$ at pH 2 compared with at $\mathrm{pH} 3$ or 9 [78]. Therefore, a decrease in membrane elasticity can reduce the endocytic ability of the cell. As for salt concentration, $\mathrm{NaCl}$ and $\mathrm{CaCl}_{2}$ can significantly increase the order of lipid molecules, leading to reduced membrane elasticity [79]. The strong interactions between PC membranes and $\mathrm{Na}^{+}$ions caused notable membrane compression, whereas the interaction of $\mathrm{K}^{+}$ions with the membrane was much weaker [81].

\section{Mathematical \& numerical modeling approaches for NM-biomembrane interactions}

Computer simulation can describe the trajectories of molecules and the dynamic process of the NM-biomembrane interactions, providing deep insights into the understanding of NM-biomembrane interactions at the nanoscale. Two types of dynamic models exist in the computer simulation model of NM-biomembrane interactions, namely the atomistic and coarse-grained models. The main difference between the two models is the simulation units that are adopted. Atomistic models employ 
simulation units of a single atom, providing a detailed description of atom-atom interactions, whereas the simulation units of coarse-grained models include a cluster of atoms, molecules or chemical groups. This section reviews the recent advances in these modeling methods, and focuses on the advantages and disadvantages of each method (Table 2).

\section{- Molecular dynamics method}

The molecular dynamics (MD) method is based on the classical Newton's law of motion. A trajectory that describes the change in an atom's position with time can be obtained by solving the differential equations of Newton's second law, with the potential energy of the atoms represented by the force field. The force field contains bond interactions that represent both the chemical bond (i.e., bond stretching, angle bending and torsion angle potentials) and nonbonded interactions (i.e., Van der Waals and electrostatic interactions). The potential energy of the system may be given as follows:

$$
V\left(r_{1}, r_{2}, \ldots r_{N}\right)=\sum_{i=1}^{N} \sum \Phi\left(r_{i j}\right)
$$

where $r_{i}$ is the location of atom $i$ and $\Phi\left(r_{i j}\right)$ is the interaction potential between atom $i$ and atom $j$. The control equations are:

$$
\begin{aligned}
& F_{i}=-\nabla_{r i}\left(r_{1}, r_{2}, \ldots r_{N}\right) \\
& m_{i} \ddot{\mathrm{r}}=F_{i}=\sum_{j \neq i} F_{i j}
\end{aligned}
$$

Numerous studies involving the MD method have been performed to determine the interactions between the biomembrane and $\mathrm{C}_{60} \mathrm{NMs}$ with different properties (i.e., morphology and concentration). The permeability coefficient had a decreasing trend as follows: nano $\mathrm{C}_{60}$, $\mathrm{C}_{60}$ and open- $\mathrm{C}_{60}$ [82]. The interactions of nine $\mathrm{C}_{60}$ molecules with the lipid bilayer were simulated to investigate the effect of a $\mathrm{C}_{60}$ concentrated solution. The results show that $\mathrm{C}_{60}$ molecules preferred to spread homogeneously near the membrane center when inserted into the membrane [83]. The preferred position, which minimized the potential of mean force, of a $\mathrm{C}_{60}$ inside a liquid crystalline lipid bilayer was approximately $6-7 \AA$ from the center of the bilayer [84]. For the more ordered gel-phase lipid bilayers, the position was approximately $18 \AA$ from the bilayer center, because of the energy penalty [85]. The permeability of $\mathrm{C}_{60}$ through the lipid bilayer correlated with its dispersion interaction with the surrounding medium [86]. The MD method was also used to investigate small molecules (e.g., benzocaine, ethanol, urea and caffeine) that penetrate across the lipid bilayer $[75,87,88]$.

The MD method is capable of capturing the details of atomic interactions. With the MD method, researchers can investigate structure dynamics changes, both for NMs and lipid molecules (e.g., the interactions between atoms in individual lipid molecules and different atom sizes of G3-PAMAM dendrimer) [89]. The atomic interactions can help us to understand interactions from the microscopic point of view, which is challenging for experimental observation. However, with present computing power, the MD method is limited by the small length and time scale (nanometer and nanosecond); hence, it is hard to simulate the endocytosis processes and large NM-biomembrane systems, which contain thousands of lipid molecules (Table 2), using this method.

\section{Coarse-grained MD method}

Compared with the MD method, the atoms are grouped together into clusters as simulation units in the coarse-grained MD (CGMD) method which will reduce computation consumption greatly. The MARTINI force field developed by Marrink et al. is a typical coarsegrained force field which is in agreement with experimental results [90]. It is widely used in biosystem simulation.

The CGMD method has been widely employed to simulate the effects of NM properties, environments and membrane properties on NM-biomembrane interactions. For example, Gu et al. simulated the effect of the hydrophobic property of NMs and the effect of charges on NM-biomembrane systems [70,91]. Hydrophobic interaction and electrostatic attraction improved the interaction process. Energy-independent endocytosis of NMs with varying shapes and ligand coverage (FIGURe 4) was simulated by Vácha $e t$ al. who indicated that endocytosis efficiency was higher for spherocylindrical NMs than for spheres [92]. As for the effect of $\mathrm{pH}$, PAMAM dendrimers can be absorbed onto the negatively charged membrane at a low $\mathrm{pH}$ and can even induce hole formation in the membrane when the corresponding protonation levels are employed in the simulation to mimic the different $\mathrm{pH}$ conditions (e.g., $\mathrm{pH} \mathrm{10,7}$ and 5) [93,94]. Aside from $\mathrm{pH}$, the formation of holes induced by Au-NMs and PAMAM dendrimers was also 
REVIEW Qu, He, Lin et al.






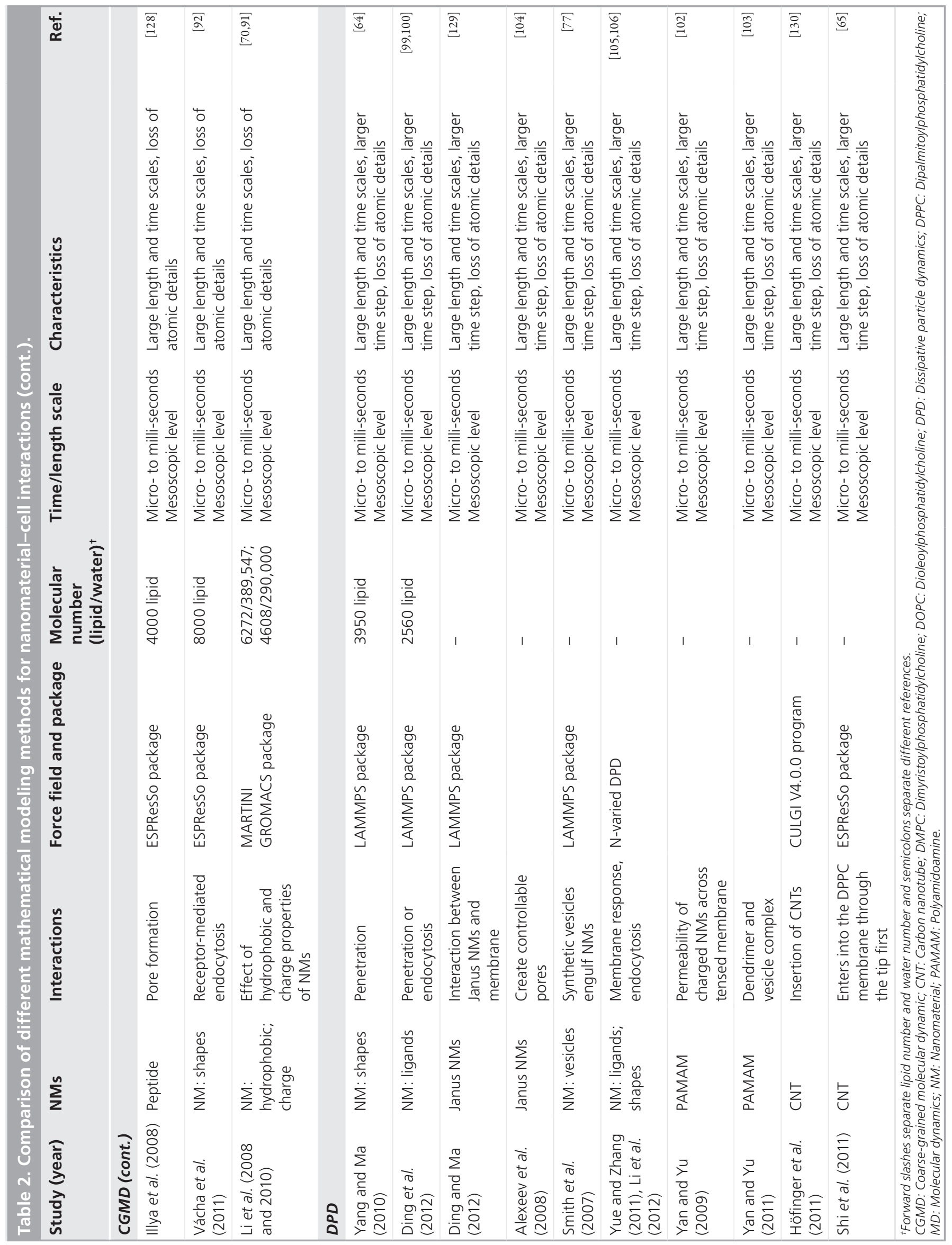



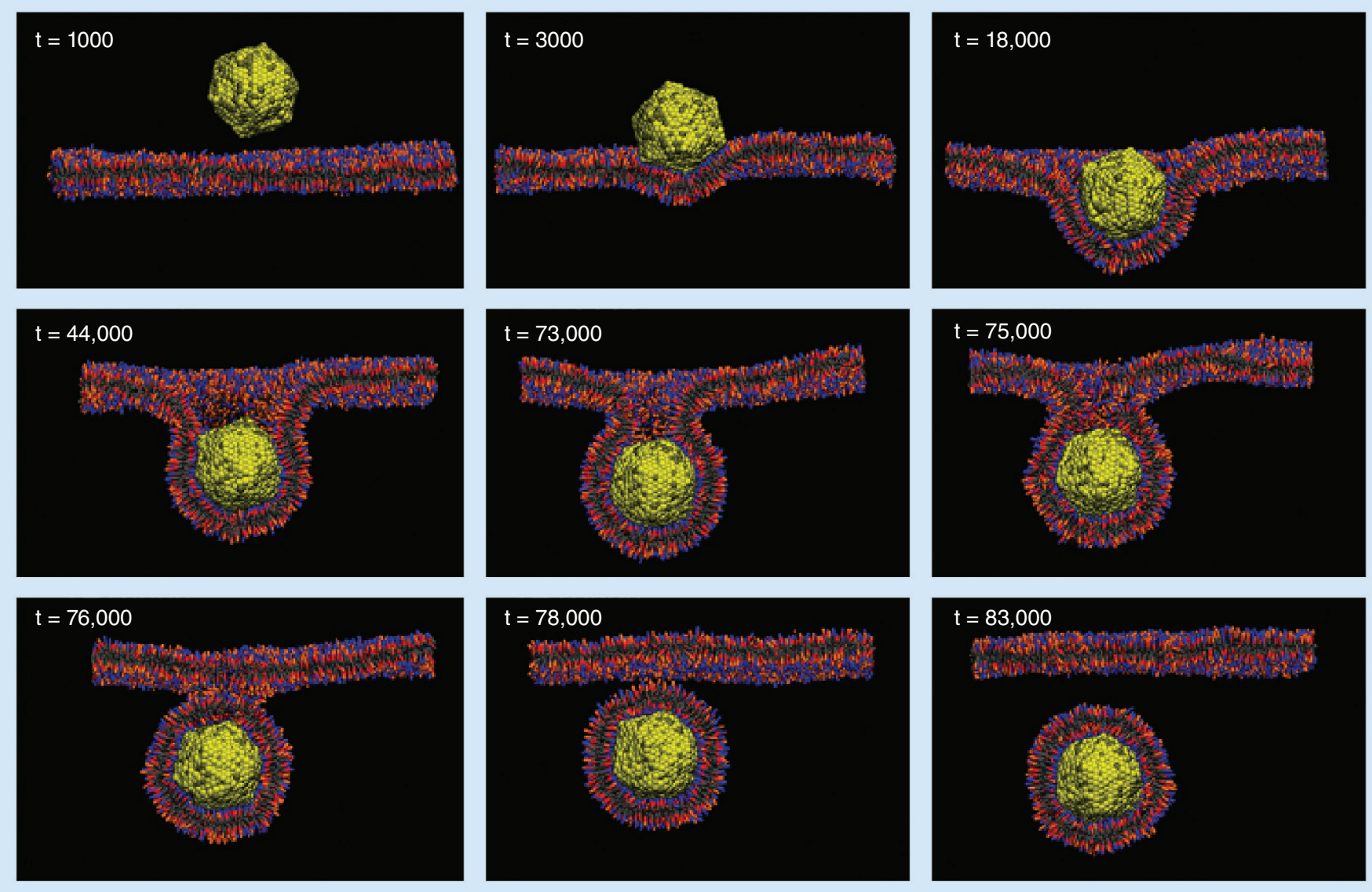

Figure 4. Snapshots of a spherical nanoparticle that underwent endocytosis by a lipid membrane. Yellow beads represent ligands. Blue beads represent membrane receptors, orange beads stand for head groups, while gray and orange are tail beads. Gray beads are purely repulsive.

Reproduced with permission from [92].

affected by surface charge signs and density of NMs, membrane surface tension and temperature $[69,95,96]$.

Compared with the MD method, the CGMD method has a longer time (millisecond) and length scale (micrometer), as shown in TABLE 2. Therefore, more experimental phenomena can be simulated by this method, such as the whole dynamic process of direct penetration and endocytosis. For instance, Figure 5 illustrates a single fullerene or a cluster of ten fullerenes penetrating the dioleoylphosphatidylcholine membrane [97]. The results are consistent with the MD simulations [83], in which the interactions were divided into two segments: the fullerenes lying inside or outside of the membrane. The permeation process of fullerene clusters into the lipid bilayer occurs on a microsecond timescale, which is too large for MD simulations to capture. Although the CGMD method loses the atomic details, acceptable simulation results can be achieved because the atom description has limited influence on the mesoscopic system [27].
Dissipative particle dynamics method The dissipative particle dynamics (DPD) method is also a coarse-grained model where individual molecules or atoms are lumped together into a 'bead'. The motion of the bead obeys Newton's equation, but each DPD bead is subjected to three types of forces, namely, conservative-repulsive forces, dissipative forces and random forces [98]. The dissipative forces and random forces are coupled through the fluctuation-dissipation theorem, and the system momentum is locally conserved.

Many complicated NM-biomembrane systems can be simulated using the DPD method. For ligand-coated NMs, a study has designed novel NMs with ligand-decorated surfaces through dynamic bonds and simulated its penetrating process through the membrane using the kinetic theory [99]:

$$
A+B \underset{k_{\text {off }}}{\stackrel{k_{o n}}{\rightleftarrows}} A B
$$

where $A$ represents NMs, $B$ represents free ligands, and $A B$ stands for the NM-ligand 
complex. The bond is broken when the bond length between the NMs and the ligand head is longer than its initial length. The bond forms again if the length is shorter than its initial length. The study provided a new way of translocating NMs and designing ligand-coated NMs. Another study investigated the effect of ligand properties (e.g., ligand density, length and rigidity) in receptor-mediated endocytosis, and found that the engulfment degree (partial or full engulfment) was strongly affected by ligand properties [100].
Coulombic interactions are needed to simulate the interaction of charged NMs with the biomembrane. Groot proposed a method based on the local lattice grid to calculate the electrostatic force [101]. Based on this method, many studies have investigated the interaction between the charged dendrimer and the membranes of different structures, such as the vesicle membrane $[102,103]$. Figure 6 shows snapshots of hole formation induced by the charged dendrimers in the tensed bilayer membrane. The figure shows that the permeability of the

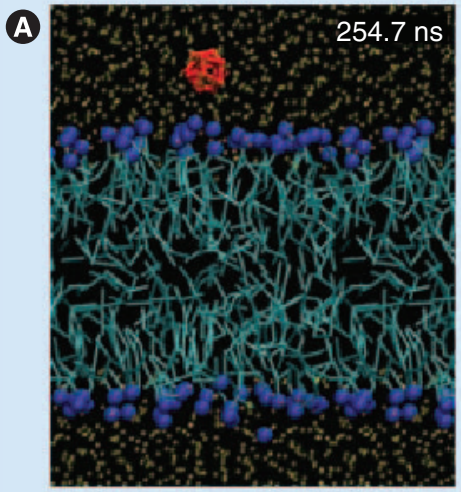

B
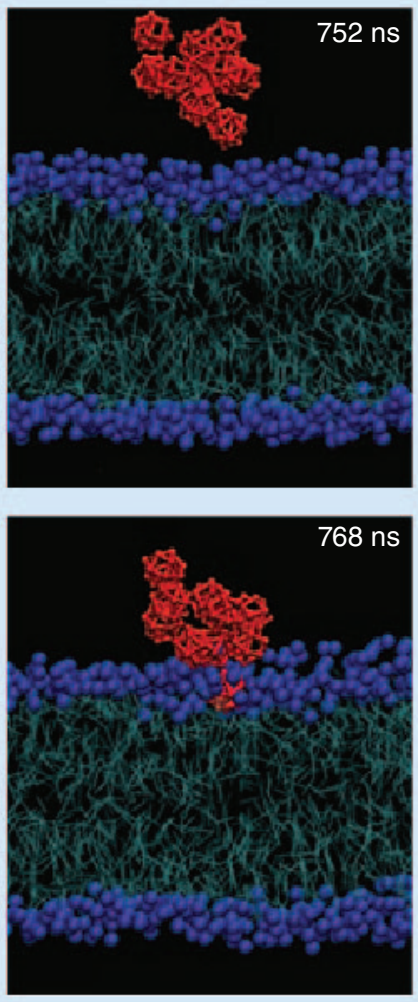
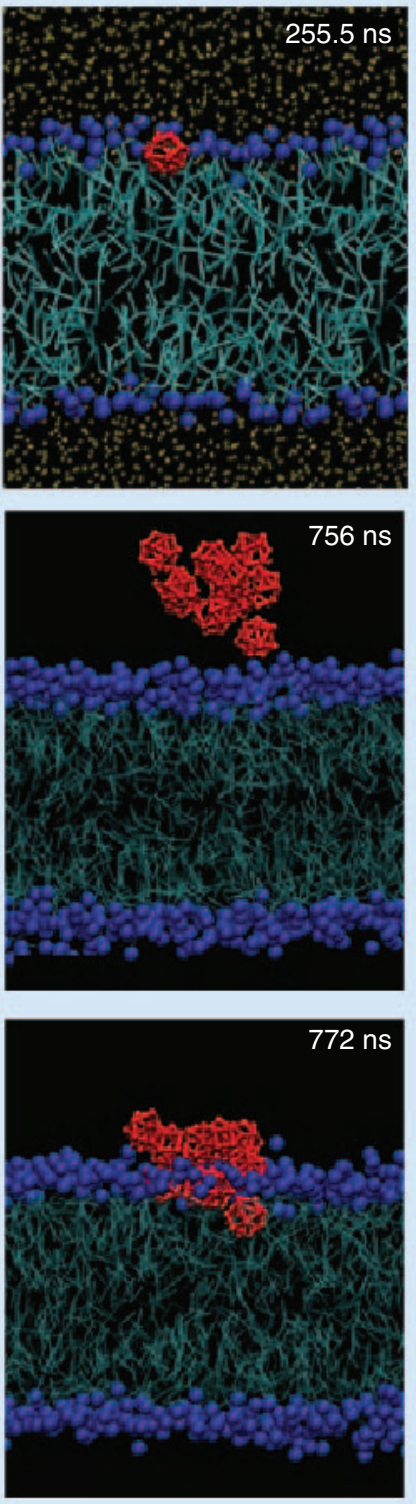
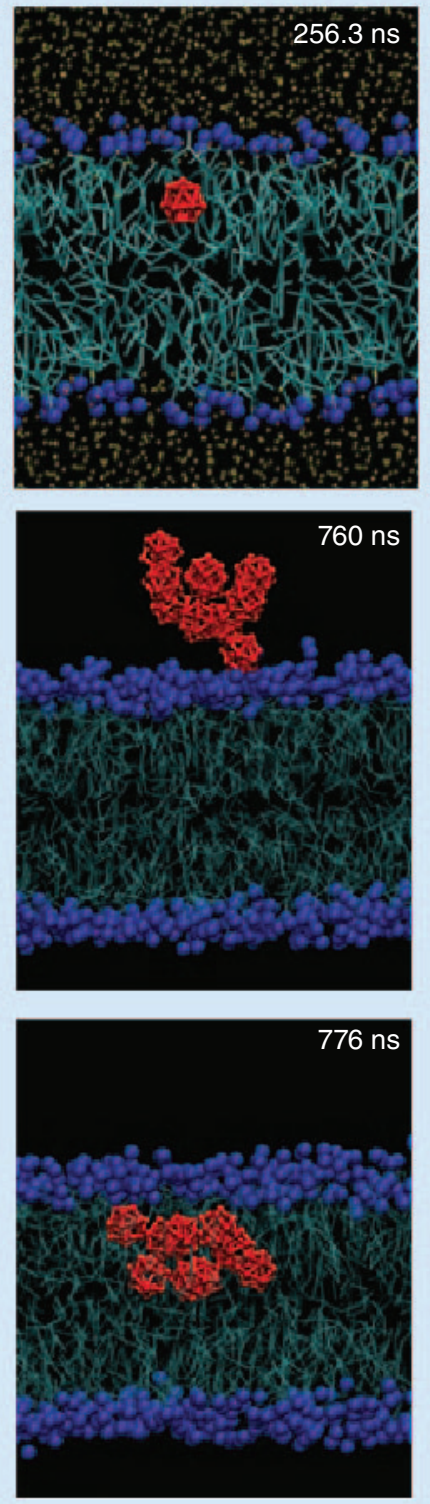
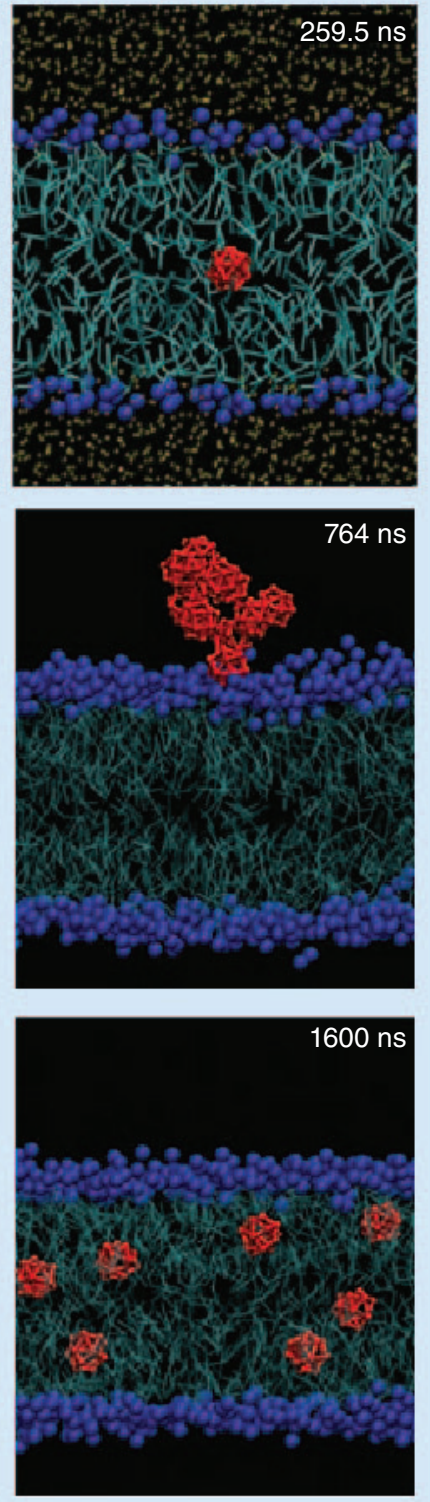

Figure 5. Penetration of a single fullerene or a cluster of ten fullerenes into the dioleoylphosphatidylcholine membrane. (A) Penetration of a single fullerene into the dioleoylphosphatidylcholine membrane. Fullerene is shown in red, the lipids in cyan with blue head groups, and water in yellow. (B) Penetration of a cluster of ten fullerenes into the dioleoylphosphatidylcholine membrane. The penetration of the fullerene cluster was much slower than a single fullerene and started with the insertion of a single fullerene in the lipid head region. Water is not represented.

Reproduced with permission from [97]. 

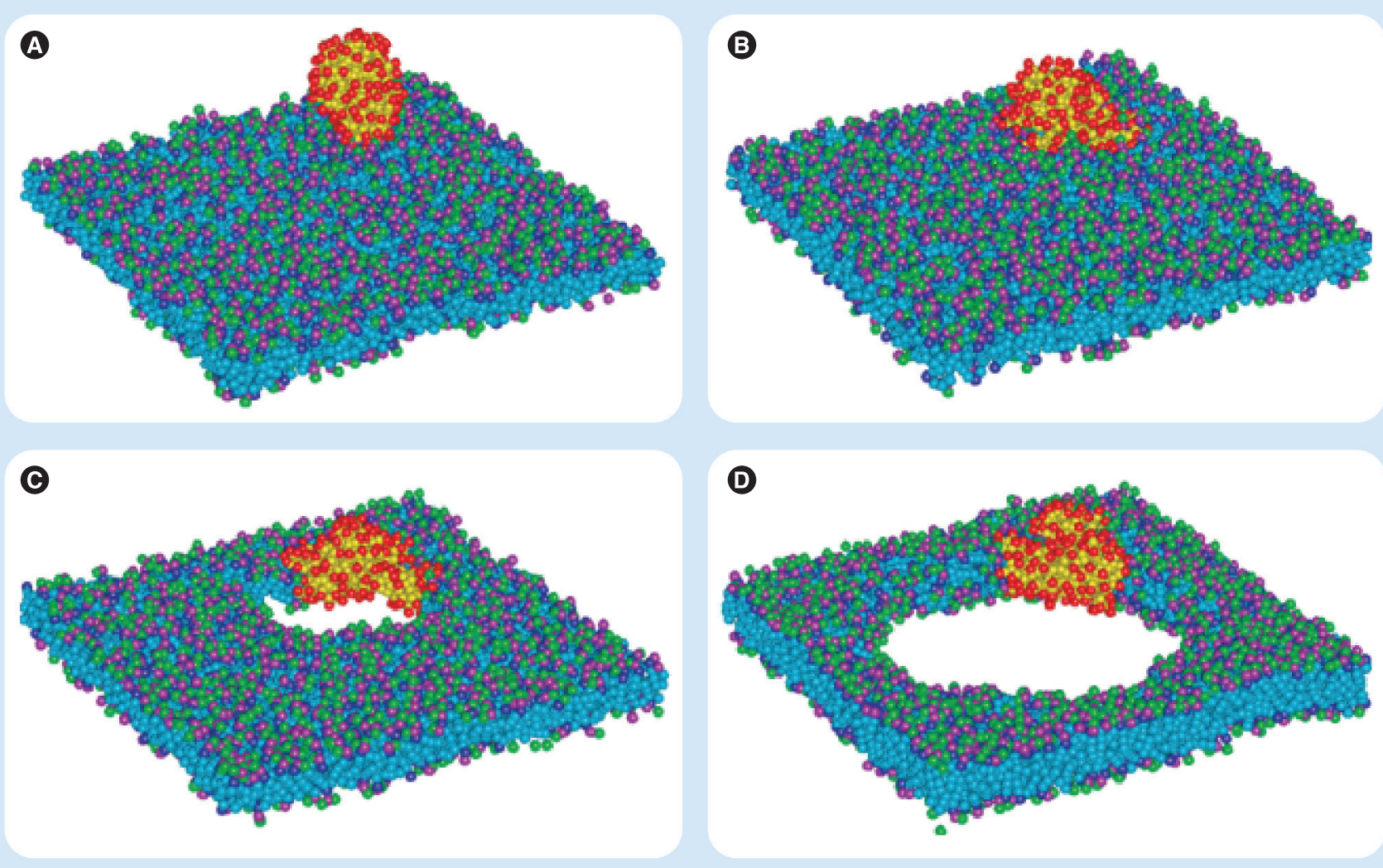

Figure 6. Snapshots of a charged G5 dendrimer-induced hole in the tense bilayer membrane. The time for each snapshot was (A) 0, (B) 4.5, (C) 6.9 and (D) $15 \mu \mathrm{s}$. Color scheme: positively charged head beads are green; negatively charged head beads are pink; uncharged head beads are blue; tail beads are cyan; charged beads of the dendrimer are red; and uncharged beads of the dendrimer are yellow. Solvent and counterions are not represented.

Reproduced with permission from [102].

charged dendrimers could be enhanced in the tensed membrane.

To simulate different membrane structures or surface tension, the bilayer membranes can be stretched by changing the lateral dimensions of the simulation box, in other words:

$$
(x, y, z) \rightarrow\left(\delta x, \delta y, \frac{z}{\delta^{2}}\right)
$$

to form pores or change surface tension in the membrane $[102,104]$. The N-varied DPD simulation with an adjusted bead number can be applied to release the tension induced by the membrane deformation, as well as to preset membrane tension and simulate the effect on the cytoskeleton and actin. This method has been employed to investigate the membrane response to the adsorption of ligand-coated NMs [105] and the endocytosis kinetics of ligand-coated NMs with different shapes [106].

Similar to the CGMD method, the DPD method has a time (millisecond) and length (micrometer) scale that allows simulation of the whole direct penetration process, the endocytosis process and other more complicated systems, such as NM-heterogeneous membrane interactions [77]. Furthermore, the DPD method allows larger time steps than the CGMD method, which greatly reduces computational efforts. In addition, the DPD method is able to describe the hydrodynamic effects because the momentum involved is conserved [107]. In addition, as with the CGMD method, the DPD method loses atomic details that cannot be used for structure analysis in the microscopic scale.

\section{Other methods}

Aside from the three methods stated above, other methods have also been used to simulate NM-biomembrane interactions, such as the Brownian dynamics method, the Monte Carlo method, thermodynamic models and mathematical models.

The Brownian dynamics method is based on the Langevin equation, where explicit solvent molecules are replaced by stochastic forces [27]. This method can simulate the phenomena with longer length (micrometer) and time scales (seconds) than the CGMD and DPD methods. For 
instance, the Brownian dynamics method was used to simulate the interactions between the lipid bilayer vesicles and adhesive NMs in which the NMs could induce vesicle budding, connection and fission [108]. The Monte Carlo method relies on repeated random sampling to obtain numerical results and it is used to model phenomena with significant uncertainty regarding inputs. For example, the Monte Carlo method was used to model NM surface rearrangements, which is important in nonspecific interactions [109]. In addition, a thermodynamic model has also been developed to investigate the condition in which the NMs could be adsorbed onto or disrupt the membrane. In this model, only the thermodynamic aspect information can be obtained without the dynamic process [110]. In contrast to molecular modeling simulations, Lunov et al. proposed a mathematic model to investigate the receptor-mediated endocytosis of engineered NPs by macrophages, which showed key parameters of this process (e.g., rate of uptake, the number of NPs per cell in saturation and the mean uptake time) [111]. They presented the understanding of $\mathrm{NM}$-cell interactions in a more macroscopic way than the dynamic method.

\section{Discussion}

Despite extensive research on the mathematical and numerical simulations of NM-biomembrane interactions, a significant discrepancy exists between the experimental observation and simulation. For instance, in a nanotube-mouse liver cell interaction experiment, the cells were exposed to CNTs for $2 \mathrm{~h}$ before observation [65]. However, in the simulation, the CNT-lipid bilayer interaction lasted for a few microseconds. The reason for this discrepancy is that it takes a long time for the CNTs to get close to the membrane surface, while we placed the CNTs on the lipid bilayer surface in the simulation. In addition, in the process of NMs getting close to the membrane surface, the protein corona and environment can also affect the NMs' properties, as discussed in the 'Key factors for NM-biomembrane interactions' section. It is challenging to observe the dynamic process of NM-biomembrane interactions in the appropriate time (microseconds) and length (several nanometers) scales. The invention of real-time monitoring microscopy with high resolution will greatly improve experimental studies. Meanwhile, for the simulation, it is difficult to take these factors into account because the simulation system within recent calculation capacity is only a few nanometers cubed, and only the lipid molecules, simplified proteins and solvents are simulated. Therefore, specific assumptions of the models are not realistic and further improvements are needed. Figure 7 shows the length and time scale for experiments and simulations. Taвle 2 summarizes the advantages and disadvantages of the main existing dynamic methods for simulating NM-cell interactions.

\section{Conclusion \& future perspective}

This article mainly reviews the cellular uptake pathways of NMs, the key factors of NMbiomembrane interactions, and the mathematical and numerical methods for NM-biomembrane interactions. The advantages and limitations for each method in terms of time and length scales were highlighted, and which proper models can be chosen to simulate different phenomena were determined. Despite the extensive achievements in this important and fast-growing field, many challenges remain. The major topics for further studies are, therefore, identified as follows:

- The membrane structure is complex in vivo because numerous proteins, sugars and cholesterol are attached to the membrane. However, the existing simulations only give a simple description of the lipid membrane skeleton, which consists of lipid molecules and simplified proteins. Simulation of more realistic lipid membranes to interact with NMs is needed;

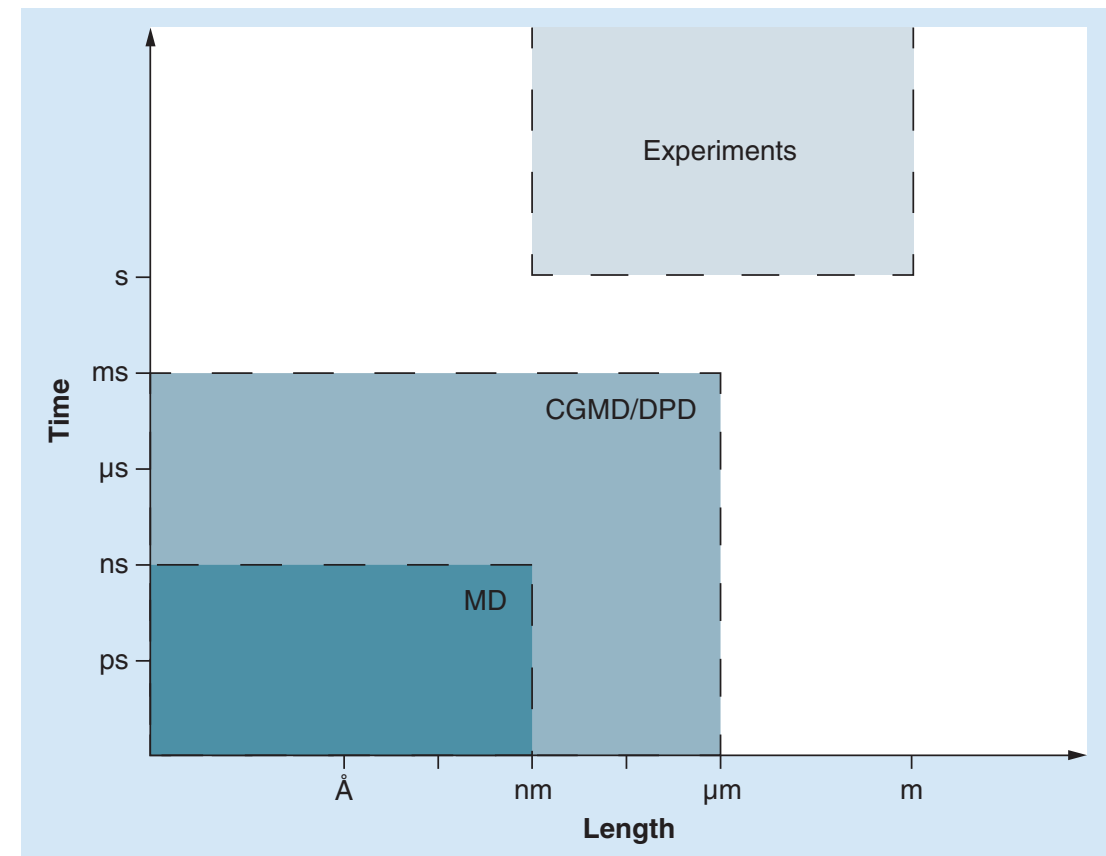

Figure 7. The length and time scale for experiments and simulations. CGMD: Coarse-grained molecular dynamics; DPD: Dissipative particle dynamics; MD: Molecular dynamics. 
- The microenvironments for various cells are different, which affects the NM-biomembrane interactions. More work is needed to match the physiological situation more closely;

- The potential functions that describe the forces between simulation units are not precise. Searching for more precise and simpler potential functions is also a challenge for simulating NM-biomembrane simulations;

- Improvement of the computer calculation capacity is needed. Simulation of large and complicated NM-biomembrane systems with a long simulated time that matches the real experiment system more closely is another challenge.

\section{Financial \& competing interests disclosure}

This work was partially supported by the National Natural Science Foundation of China (10825210, 31050110125 and 11272327) and the National 111 Project of China (B06024). FXu was also partially supported by the China Young 1000-Talent Program and Shaanxi 100-Talent Program, Program for New Century Excellent Talents in University (NCET-12-0437), and International S\&T Cooperation Program of China (2013DFG02930). The authors have no other relevant affiliations or financial involvement with any organization or entity with a financial interest in or financial conflict with the subject matter or materials discussed in the manuscript apart from those disclosed.

No writing assistance was utilized in the production of this manuscript.

\section{Executive summary}

- Fundamental studies are necessary in order to obtain a systematic understanding of the mechanism underlying nanomaterial (NM)-biomembrane interactions. Mathematical and numerical modeling can provide deep insight into the dynamic process of the system, which has been demonstrated to be a useful tool.

- The cellular uptake of various NMs generally includes endocytic and nonendocytic pathways.

- Key factors that affect NM-biomembrane interactions include physicochemical properties of NMs (size, shape, surface roughness and rigidity, charge, hydrophobic properties and protein corona), biomembrane properties, temperature, $\mathrm{pH}$ and salt concentration.

- There are two types of dynamic model for the simulation of NM-biomembrane interactions, namely, atomistic models (molecular dynamics) and coarse-grained models (coarse-grained molecular dynamics and dissipative particle dynamics). The length and time scales of each method determine the simulated system phenomena.

- Simulation of a larger NM-biomembrane interaction system that matches the physiological situation more closely is needed in future studies.

\section{References}

Papers of special note have been highlighted as:

- of interest

1 Nohynek GJ, Lademann J, Ribaud C, Roberts MS. Grey goo on the skin? Nanotechnology, cosmetic and sunscreen safety. Crit. Rev. Toxicol. 37(3), 251-277 (2007).

2 Dastjerdi R, Montazer M. A review on the application of inorganic nano-structured materials in the modification of textiles: focus on anti-microbial properties. Colloids Surf. $B$ 79(1), 5-18 (2010).

3 El-Sayed IH, Huang X, El-Sayed MA. Surface plasmon resonance scattering and absorption of anti-EGFR antibody conjugated gold nanoparticles in cancer diagnostics: applications in oral cancer. Nano Lett. 5(5), 829-834 (2005).

4 Freitas Jr RA. What is nanomedicine? Nanomedicine 1(1), 2-9 (2005).

5 Campolongo MJ, Tan SJ, Xu J, Luo D. DNA nanomedicine: engineering DNA as a polymer for therapeutic and diagnostic applications. Adv. Drug Deliv. Rev, 62(6), 606-616 (2010).

6 Lin M, Zhao Y, Wang S et al. Recent advances in synthesis and surface modification of lanthanide-doped upconversion nanoparticles for biomedical applications. Biotechnol. Adv. 30(6), 1551-1561 (2012).

7 Sha B, Wei G, Han Y et al. Potential application of titanium dioxide nanoparticles in the prevention of osteosarcoma and chondrosarcoma recurrence. J. Nanosci. Nanotechnol. 12, 1-4 (2012).

8 Baoyong S, Gao W, Wang S, Xu F, Lu T. Cytotoxicity of titanium dioxide nanoparticles differs in four liver cells from human and rat. Composites Part B 42(8), 2136-2144 (2011).

9 Song Y, Li X, Du X. Exposure to nanoparticles is related to pleural effusion, pulmonary fibrosis and granuloma. Eur. Respir. J. 34(3), 559-567 (2009).

10 Tinkle SS, Antonini JM, Rich BA et al. Skin as a route of exposure and sensitization in chronic beryllium disease. Environ. Health Perspect. 111(9), 1202-1208 (2003).

11 Gontier E, Ynsa MD, Biro T et al. Is there penetration of titania nanoparticles in sunscreens through skin? A comparative electron and ion microscopy study. Nanotoxicology 2(4), 218-231 (2008).
12 Muller RH, Maassen S, Schwarz C, Mehnert W. Solid lipid nanoparticles (SLN) as potential carrier for human use: interaction with human granulocytes. J. Control. Release 47(3), 261-269 (1997).

13 Yang WE, Hsu ML, Lin MC, Chen ZH, Chen LK, Huang HH. Nano/submicronscale $\mathrm{TiO}_{2}$ network on titanium surface for dental implant application. J. Alloys Compd 479(1-2), 642-647 (2009).

14 Lewinski N, Colvin V, Drezek R. Cytotoxicity of nanoparticles. Small, 4(1), 26-49 (2008).

15 Zhao F, Zhao Y, Liu Y, Chang X, Chen C, Zhao Y. Cellular uptake, intracellular trafficking, and cytotoxicity of nanomaterials. Small 7(10), 1322-1337 (2011).

16 Monticelli L, Salonen E, Ke PC, Vattulainen I. Effects of carbon nanoparticles on lipid membranes: a molecular simulation perspective. Soft Matter 5(22), 4433 (2009).

17 Wilhelm C, Billotey C, Roger J, Pons JN, Bacri JC, Gazeau F. Intracellular uptake of anionic superparamagnetic nanoparticles as a function of their surface coating. Biomaterials 24(6), 1001-1011 (2003). 
18 Li SQ, Zhu RR, Zhu H et al. Nanotoxicity of $\mathrm{TiO}_{2}$ nanoparticles to erythrocyte in vitro. Food Chem. Toxicol. 46(12), 3626-3631 (2008).

19 Leroueil PR, Berry SA, Duthie K et al. Wide varieties of cationic nanoparticles induce defects in supported lipid bilayers. Nano Lett. 8(2), 420-424 (2008).

20 Isakovic A, Markovic Z, Todorovic-Markovic B et al. Distinct cytotoxic mechanisms of pristine versus hydroxylated fullerene. Toxicol. Sci. 91(1), 173-183 (2006).

21 Vevers WF, Jha AN. Genotoxic and cytotoxic potential of titanium dioxide $(\mathrm{TiO} 2)$ nanoparticles on fish cells in vitro. Ecotoxicology 17(5), 410-420 (2008).

22 Gul-Uludag H, Lu W, Xu P, Xing J, Chen J. Efficient and rapid uptake of magnetic carbon nanotubes into human monocytic cells: implications for cell-based cancer gene therapy. Biotechnol. Lett. 34(5), 989-993 (2012).

23 Zhao L, Wu J, Zhou $\mathrm{H}$ et al. Local gene delivery for cancer therapy. Curr. Gene Ther. 11(5), 423-432 (2011).

24 Kirui DK, Rey DA, Batt CA. Gold hybrid nanoparticles for targeted phototherapy and cancer imaging. Nanotechnology 21(10), 105105 (2012).

25 Pissuwan D, Niidome T, Cortie MB. The forthcoming applications of gold nanoparticles in drug and gene delivery systems. J. Control. Release 149(1), 65-71 (2011).

26 Han G, Ghosh P, Rotello VM. Functionalized gold nanoparticles for drug delivery. Nanomedicine (Lond.) 2(1), 113-123 (2007).

- Discusses recent advances in the drug delivery field using gold nanoparticles as carriers for therapeutic agents. Drug delivery is one of the most important fields that nanomaterial-biomembrane interactions are considered in.

27 Shillcock JC. Insight or illusion? Seeing inside the cell with mesoscopic simulations. HFSP J. 2(1), 1-6 (2008).

28 Venturoli M, Maddalena Sperotto M, Kranenburg M, Smit B. Mesoscopic models of biological membranes. Phys. Rep. 437(1-2), 1-54 (2006).

29 Makarucha A, Todorova N, Yarovsky I. Nanomaterials in biological environment: a review of computer modelling studies. Eur. Biophys. J. 40 (2), 103-115 (2011).

30 Zhang Y, Yang M, Portney N et al. Zeta potential: a surface electrical characteristic to probe the interaction of nanoparticles with normal and cancer human breast epithelial cells. Biomed. Microdevices 10(2), 321-328 (2008).
31 Leroueil PR, Hong S, Mecke A, Baker JR, Orr BG, Banaszak Holl MM. Nanoparticle interaction with biological membranes: does nanotechnology present a Janus face? Acc. Chem. Res. 40(5), 335-342 (2007).

32 Geiser M, Rothen-Rutishauser B, Kapp N et al. Ultrafine particles cross cellular membranes by nonphagocytic mechanisms in lungs and in cultured cells. Environ. Health Perspect. 113(11), 1555-1560 (2005).

33 Kievit FM, Zhang M. Cancer nanotheranostics: improving imaging and therapy by targeted delivery across biological barriers. Adv. Mater. 23(36), H217-H247 (2011).

34 Hillaireau H, Couvreur P. Nanocarriers' entry into the cell: relevance to drug delivery. Cell. Mol. Life Sci. 66(17), 2873-2896 (2009).

35 Verma A, Stellacci F. Effect of surface properties on nanoparticle-cell interactions. Small 6(1), 12-21 (2010).

36 Chou LYT, Ming K, Chan WCW. Strategies for the intracellular delivery of nanoparticles. Chem. Soc. Rev. 40(1), 233-245 (2011).

37 Chithrani BD, Ghazani AA, Chan WCW. determining the size and shape dependence of gold nanoparticle uptake into mammalian cells. Nano Lett. 6(4), 662-668 (2006).

38 Chithrani BD, Chan WCW. Elucidating the mechanism of cellular uptake and removal of protein-coated gold nanoparticles of different sizes and shapes. Nano Lett. 7(6), 1542-1550 (2007).

39 Kam NWS, Jessop TC, Wender PA, Dai H. Nanotube molecular transporters: internalization of carbon nanotube-protein conjugates into mammalian cells. J. Am. Chem. Soc. 126(22), 6850-6851 (2004).

40 Kam NWS, Liu Z, Dai H. Carbon nanotubes as intracellular transporters for proteins and DNA: an investigation of the uptake mechanism and pathway. Angew. Chem. Int. Ed. Engl. 118(4), 591-595 (2006).

41 Oh J-M, Choi S-J, Kim S-T, Choy J-H. Cellular uptake mechanism of an inorganic nanovehicle and its drug conjugates: enhanced efficacy due to clathrin-mediated endocytosis. Bioconjug. Chem. 17(6), 1411-1417 (2006).

42 Zhang LW, Monteiro-Riviere NA. Mechanisms of quantum dot nanoparticle cellular uptake. Toxicol. Sci. 110(1), 138-155 (2009).

43 Corot C, Petry KG, Trivedi R et al. Macrophage imaging in central nervous system and in carotid atherosclerotic plaque using ultrasmall superparamagnetic iron oxide in magnetic resonance imaging. Invest. Radiol. 39(10), 619-625 (2004).
44 Wang Y, Li B, Xu F, Jia D, Feng Y, Zhou Y In vitro cell uptake of biocompatible magnetite/chitosan nanoparticles with high magnetization: a single-step synthesis approach for $i n$-situ-modified magnetite by amino groups of chitosan. J. Biomater. Sci. Polym. Ed. 23(7), 843-860 (2012).

45 Nel AE, Madler L, Velegol D et al. Understanding biophysicochemical interactions at the nano-bio interface. Nat. Mater. 8(7), 543-557 (2009).

- Reviews the biophysicochemical interactions at the nano-biomembrane interface, including nanoparticles interacting with proteins, membranes, cells, DNA and organelles. Helpful for understanding the background of nanomaterial-biomembrane interactions.

46 Zhang S, Li J, Lykotrafitis G, Bao G, Suresh S Size-dependent endocytosis of nanoparticles. Adv. Mater. 21(4), 419-424 (2009).

47 Gao H, Shi W, Freund LB. Mechanics of receptor-mediated endocytosis. Proc. Natl Acad. Sci. USA 102(27), 9469-9474 (2005).

48 Zhao Y, Sun X, Zhang G, Trewyn BG, Slowing II, Lin VSY. Interaction of mesoporous silica nanoparticles with human red blood cell membranes: size and surface effects. ACS Nano 5(2), 1366-1375 (2011).

$49 \mathrm{Yu}$ J, Patel SA, Dickson RM. In vitro and intracellular production of peptideencapsulated fluorescent silver nanoclusters. Angew. Chem. Int. Ed. Engl. 119(12), 2074-2076 (2007).

$50 \mathrm{Mu}$ Q, Broughton DL, Yan B. Endosomal leakage and nuclear translocation of multiwalled carbon nanotubes: developing a model for cell uptake. Nano Lett. 9(12), 4370-4375 (2009).

51 Hong S, Leroueil PR, Janus EK et al. Interaction of polycationic polymers with supported lipid bilayers and cells: nanoscale hole formation and enhanced membrane permeability. Bioconjug. Chem. 17(3), 728-734 (2006).

52 Hong S, Bielinska AU, Mecke A et al. Interaction of poly(amidoamine) dendrimers with supported lipid bilayers and cells: hole formation and the relation to transport. Bioconjug. Chem. 15(4), 774-782 (2004).

53 Foerg C, Merkle HP. On the biomedical promise of cell penetrating peptides: limits versus prospects. J. Pharm. Sci. 97(1), 144-162 (2008).

54 Sebbage V. Cell-penetrating peptides and their therapeutic applications. Bio. Horizons 2(1), 64-72 (2009).

55 Veiseh O, Kievit FM, Mok H et al. Cell transcytosing poly-arginine coated magnetic nanovector for safe and effective siRNA 
delivery. Biomaterials 32(24), 5717-5725 (2011).

56 Goda T, Goto Y, Ishihara K. Cell-penetrating macromolecules: direct penetration of amphipathic phospholipid polymers across plasma membrane of living cells. Biomaterials 31(8), 2380-2387 (2010).

57 Verma A, Uzun O, Hu Y et al. Surfacestructure-regulated cell-membrane penetration by monolayer-protected nanoparticles. Nat. Mater. 7(7), 588-595 (2008).

58 Li Y, Li X, Li Z, Gao H. Surface-structureregulated penetration of nanoparticles across a cell membrane. Nanoscale 4(12), 3768-3775 (2012).

59 Roiter Y, Ornatska M, Rammohan AR, Balakrishnan J, Heine DR, Minko S. Interaction of nanoparticles with lipid membrane. Nano Lett. 8(3), 941-944 (2008).

60 Roiter Y, Ornatska M, Rammohan AR, Balakrishnan J, Heine DR, Minko S. Interaction of lipid membrane with nanostructured surfaces. Langmuir 25(11), 6287-6299 (2009).

61 Jiang W, Kim BY, Rutka JT, Chan WC. Nanoparticle-mediated cellular response is size-dependent. Nat. Nanotechnol. 3(3), 145-150 (2008).

62 Champion JA, Mitragotri S. Role of target geometry in phagocytosis. Proc. Natl Acad. Sci. USA 103(13), 4930-4934 (2006).

63 Gratton SEA, Ropp PA, Pohlhaus PD et al. The effect of particle design on cellular internalization pathways. Proc. Natl Acad. Sci. USA 105(33), 11613-11618 (2008).

64 Yang K, Ma Y-Q. Computer simulation of the translocation of nanoparticles with different shapes across a lipid bilayer. Nat. Nanotechnol. 5(8), 579-583 (2010).

- Used the dissipative particle dynamics method to investigate nanoparticles with different shapes interacting with the lipid bilayer. Provides a practical guide to geometry considerations when designing nanoscale cargo carriers through simulation.

65 Shi X, von dem Bussche A, Hurt RH, Kane $\mathrm{AB}, \mathrm{Gao} \mathrm{H}$. Cell entry of one-dimensional nanomaterials occurs by tip recognition and rotation. Nat. Nanotechnol. 6(11), 714-719 (2011).

- Investigates cell entry of 1D nanomaterials using the coarse-grained molecular dynamics method, which is in agreement with the experimental results of the coarse-grained molecular dynamic method in nanomaterial-biomembrane interaction simulation.

66 Yi X, Shi X, Gao H. Cellular uptake of elastic nanoparticles. Phys. Rev. Lett. 107(9), 098101 (2011).
67 Sahay G, Alakhova DY, Kabanov AV. Endocytosis of nanomedicines. J. Control. Release 145(3), 182-195 (2010).

68 Goodman CM, McCusker CD, Yilmaz T, Rotello VM. Toxicity of gold nanoparticles functionalized with cationic and anionic side chains. Bioconjug. Chem. 15(4), 897-900 (2004).

69 Lin J, Zhang $\mathrm{H}$, Chen Z, Zheng Y. Penetration of lipid membranes by gold nanoparticles: insights into cellular uptake, cytotoxicity, and their relationship. ACS Nano 4(9), 5421-5429 (2010).

70 Li Y, Chen X, Gu N. Computational investigation of interaction between nanoparticles and membranes: hydrophobic/ hydrophilic effect. J. Phys. Chem. B 112(51), 16647-16653 (2008).

71 Tenzer S, Docter D, Rosfa S et al. Nanoparticle size is a critical physicochemical determinant of the human blood plasma corona: a comprehensive quantitative proteomic analysis. ACS Nano 5(9), 7155-7167 (2011).

72 Owens DE 3rd, Peppas NA. Opsonization, biodistribution, and pharmacokinetics of polymeric nanoparticles. Int. J. Pharm. 307(1), 93-102 (2006).

73 Prapainop K, Witter DP, Wentworth P. A chemical approach for cell-specific targeting of nanomaterials: small-molecule-initiated misfolding of nanoparticle corona proteins. J. Am. Chem. Soc. 134(9), 4100-4103 (2012).

74 Zhu Y, Li W, Li Q et al. Effects of serum proteins on intracellular uptake and cytotoxicity of carbon nanoparticles. Carbon 47(5), 1351-1358 (2009).

75 Porasso RD, Drew Bennett WF, Oliveira-Costa SD, López Cascales JJ. Study of the benzocaine transfer from aqueous solution to the interior of a biological membrane. J. Phys. Chem. B 113(29), 9988-9994 (2009).

76 Mecke A, Lee D-K, Ramamoorthy A, Orr BG, Banaszak Holl MM. Synthetic and natural polycationic polymer nanoparticles interact selectively with fluid-phase domains of DMPC lipid bilayers. Langmuir 21(19), 8588-8590 (2005).

77 Smith KA, Jasnow D, Balazs AC. Designing synthetic vesicles that engulf nanoscopic particles. J. Chem. Phys. 127(8), 084703 (2007)

78 Zhou Y, Raphael RM. Solution pH alters mechanical and electrical properties of phosphatidylcholine membranes: relation between interfacial electrostatics, intramembrane potential, and bending elasticity. Biophys. J. 92(7), 2451-2462 (2007).

79 Pabst G, Hodzic A, Štrancar J, Danner S, Rappolt M, Laggner P. Rigidification of neutral lipid bilayers in the presence of salts. Biophys. J. 93(8), 2688-2696 (2007).

80 Vihola H, Marttila A-K, Pakkanen JS et al. Cell-polymer interactions of fluorescent polystyrene latex particles coated with thermosensitive poly ( $N$-isopropylacrylamide) and poly $(N$-vinylcaprolactam) or grafted with poly(ethylene oxide)-macromonomer. Int . J. Pharm. 343(1-2), 238-246 (2007).

81 Gurtovenko AA, Vattulainen I. Effect of $\mathrm{NaCl}$ and $\mathrm{KCl}$ on phosphatidylcholine and phosphatidylethanolamine lipid membranes: insight from atomic-scale simulations for understanding salt-induced effects in the plasma membrane. J. Phys. Chem. B 112(7), 1953-1962 (2008).

82 Fiedler SL, Violi A. Simulation of nanoparticle permeation through a lipid membrane. Biophys. J. 99(1), 144-152 (2010).

83 Chang R, Lee J. Dynamics of $\mathrm{C}_{60}$ molecules in biological membrnaes: computer simulation studes. Bull. Korean Chem. Soc. 31(11), 3195-3200 (2010).

84 Li L, Davande H, Bedrov D, Smith GD. A molecular dynamics simulation study of $\mathrm{C}_{60}$ Fullerenes inside a dimyristoylphosphatidylcholine lipid bilayer. J. Phys. Chem. B 111(16), 4067-4072 (2007).

85 Redmill PS, McCabe C. Molecular dynamics study of the behavior of selected nanoscale building blocks in a gel-phase lipid bilayer. J. Phys. Chem. B 114(28), 9165-9172 (2010).

86 Bedrov D, Smith GD, Davande H, Li L. Passive transport of C60 fullerenes through a lipid membrane: a molecular dynamics simulation study. J. Phys. Chem. B 112(7), 2078-2084 (2008).

87 Liu J, Hopfinger AJ. Identification of possible sources of nanotoxicity from carbon nanotubes inserted into membrane bilayers using membrane interaction quantitative structure-activity relationship analysis. Chem. Res. Toxicol. 21(2), 459-466 (2008).

88 Marrink SJ, Berendsen HJC. Permeation process of small molecules across lipid membranes studied by molecular dynamics simulations. J. Phys. Chem. 100 (41), 16729-16738 (1996).

89 Kelly CV, Leroueil PR, Nett EK et al. Poly(amidoamine) dendrimers on lipid bilayers I: free energy and conformation of binding. J. Phys. Chem. B 112(31), 9337-9345 (2008).

90 Marrink SJ, de Vries AH, Mark AE. Coarse grained model for semiquantitative lipid simulations. J. Phys. Chem. B 108(2), 750-760 (2003)

91 Li Y, Gu N. Thermodynamics of charged nanoparticle adsorption on charge-neutral membranes: a simulation study. J. Phys. Chem. B 114(8), 2749-2754 (2010). 
92 Vácha R, Martinez-Veracoechea FJ, Frenkel D. Receptor-mediated endocytosis of nanoparticles of various shapes. Nano Lett. 11(12), 5391-5395 (2011).

93 Tian W-D, Ma Y-Q. pH-responsive dendrimers interacting with lipid membranes. Soft Matter 8(9), 2627-2632 (2012).

94 Tian W-D, Ma Y-Q. Insights into the endosomal escape mechanism via investigation of dendrimer-membrane interactions. Soft Matter 8, 6378-6384 (2012).

95 Lin J-Q, Zheng Y-G, Zhang H-W, Chen Z. A simulation study on nanoscale holes generated by gold nanoparticles on negative lipid bilayers. Langmuir 27(13), 8323-8332 (2011).

96 Lee H, Larson RG. Molecular dynamics simulations of PAMAM dendrimer-induced pore formation in DPPC bilayers with a coarse-grained model. J. Phys. Chem. B 110(37), 18204-18211 (2006).

97 Wong-Ekkabut J, Baoukina S, Triampo W, Tang IM, Tieleman DP, Monticelli L. Computer simulation study of fullerene translocation through lipid membranes. Nat. Nanotechnol. 3(6), 363-368 (2008).

98 Frenkel D, Smit B. Understanding Molecular Simulation: From Algorithms to Applications. Academic Press, MA, USA (2001).

99 Ding H-M, Tian W-D, Ma Y-Q. Designing nanoparticle translocation through membranes by computer simulations. ACS Nano 6(2), 1230-1238 (2012).

100 Ding H-M, Ma Y-Q. Role of physicochemical properties of coating ligands in receptormediated endocytosis of nanoparticles. Biomaterials 33(23), 5798-5802 (2012).

101 Groot R. Electrostatic interactions in dissipative particle dynamics - simulation of polyelectrolytes and anionic surfactants. J. Chem. Phys. 118, 11265 (2003).

102 Yan L-T, Yu X. Enhanced permeability of charged dendrimers across tense lipid bilayer membranes. ACS Nano 3(8), 2171-2176 (2009).

103 Yan LT, Yu X. Complexes comprised of a dendrimer and a vesicle: role of vesicle size and the surface tension of the vesicle membrane. Nanoscale 3(9), 3812-3818 (2011).

104 Alexeev A, Uspal WE, Balazs AC. Harnessing Janus nanoparticles to create controllable pores in membranes. ACS Nano 2(6), 1117-1122 (2008)

105 Yue T, Zhang X. Molecular understanding of receptor-mediated membrane responses to ligand-coated nanoparticles. Soft Matter 7(19), 9104-9112 (2011)

106 Li Y, Yue T, Yang K, Zhang X. Molecular modeling of the relationship between nanoparticle shape anisotropy and endocytosis kinetics. Biomaterials 33(19), 4965-4973 (2012).

107 Müller M, Katsov K, Schick M. Biological and synthetic membranes: what can be learned from a coarse-grained description? Phys. Rep. 434(5-6), 113-176 (2006).

108 Noguchi H, Takasu M. Adhesion of nanoparticles to vesicles: a Brownian dynamics simulation. Biophys. J. 83(1), 299-308 (2002).

109 Van Lehn RC, Alexander-Katz A. Penetration of lipid bilayers by nanoparticles with environmentally-responsive surfaces: simulations and theory. Soft Matter 7(24), 11392 (2011).

110 Ginzburg VV, Balijepalli S. Modeling the thermodynamics of the interaction of nanoparticles with cell membranes. Nano Lett. 7(12), 3716-3722 (2007)

111 Lunov O, Zablotskii V, Syrovets T et al. Modeling receptor-mediated endocytosis of polymer-functionalized iron oxide nanoparticles by human macrophages. Biomaterials 32(2), 547-555 (2011)

112 Petri-Fink A, Chastellain M, Juillerat-Jeanneret L, Ferrari A, Hofmann H. Development of functionalized superparamagnetic iron oxide nanoparticles for interaction with human cancer cells. Biomaterials 26(15), 2685-2694 (2005).

113 Qaddoumi M, Ueda H, Yang J, Davda J, Labhasetwar V, Lee V. The characteristics and mechanisms of uptake of PLGA nanoparticles in rabbit conjunctival epithelial cell layers. Pharm. Res. 21(4), 641-648 (2004).

114 Harush-Frenkel O, Debotton N, Benita S, Altschuler Y. Targeting of nanoparticles to the clathrin-mediated endocytic pathway. Biochem. Biophys. Res. Commun. 353(1), 26-32 (2007).

115 Dausend J, Musyanovych A, Dass M et al. Uptake mechanism of oppositely charged fluorescent nanoparticles in HeLa cells. Macromol. Biosci. 8(12), 1135-1143 (2008).

116 Sahay G, Kim JO, Kabanov AV, Bronich TK. The exploitation of differential endocytic pathways in normal and tumor cells in the selective targeting of nanoparticulate chemotherapeutic agents. Biomaterials 31(5), 923-933 (2010)

117 Huang X, Teng X, Chen D, Tang F, He J. The effect of the shape of mesoporous silica nanoparticles on cellular uptake and cell function. Biomaterials 31(3), 438-448 (2010).

118 Nan A, Bai X, Son SJ, Lee SB, Ghandehari H. Cellular uptake and cytotoxicity of silica nanotubes. Nano Lett. 8(8), 2150-2154 (2008).
119 Brown DM, Kinloch IA, Bangert U et al. An in vitro study of the potential of carbon nanotubes and nanofibres to induce inflammatory mediators and frustrated phagocytosis. Carbon 45(9), 1743-1756 (2007).

120 Matsuzaki K, Sugishita K-I, Ishibe N et al. Relationship of membrane curvature to the formation of pores by magainin 2 . Biochemistry 37(34), 11856-11863 (1998).

121 Cruz-Chu ER, Aksimentiev A, Schulten K. Water-silica force field for simulating nanodevices. J. Phys. Chem. B 110(43), 21497-21508 (2006).

122 Smondyrev AM, Berkowitz ML. Structure of dipalmitoylphosphatidylcholine/cholesterol bilayer at low and high cholesterol concentrations: molecular dynamics simulation. Biophys. J. 77(4), 2075-2089 (1999).

123 Smondyrev AM, Berkowitz ML. United atom force field for phospholipid membranes: constant pressure molecular dynamics simulation of dipalmitoylphosphatidicholine/ water system. J. Comput. Chem. 20(5), 531-545 (1999).

124 Berger O, Edholm O, Jähnig F. Molecular dynamics simulations of a fluid bilayer of dipalmitoylphosphatidylcholine at full hydration, constant pressure, and constant temperature. Biophys. J. 72(5), 2002-2013 (1997).

125 MacCallum JL, Tieleman DP. Computer simulation of the distribution of hexane in a lipid bilayer: spatially resolved free energy, entropy, and enthalpy profiles. J. Am. Chem. Soc. 128(1), 125-130 (2005).

126 Lee H, Larson RG. Lipid bilayer curvature and pore formation induced by charged linear polymers and dendrimers: the effect of molecular shape. J. Phys. Chem. B 112(39), 12279-12285 (2008).

127 Lee H, Larson RG. Coarse-grained molecular dynamics studies of the concentration and size dependence of fifth- and seventh-generation PAMAM dendrimers on pore formation in DMPC bilayer. J. Phys. Chem. B 112(26), 7778-7784 (2008).

128 Illya G, Deserno M. Coarse-grained simulation studies of peptide-induced pore formation. Biophys. J. 95(9), 4163-4173 (2008).

129 Ding HM, Ma YQ. Interactions between Janus particles and membranes. Nanoscale 4(4), 1116-1122 (2012).

130 Höfinger S, Melle-Franco M, Gallo T et al. A computational analysis of the insertion of carbon nanotubes into cellular membranes. Biomaterials 32(29), 7079-7085 (2011). 